\title{
Avaliação da dor em crianças com câncer: revisão narrativa de literatura
}

\author{
Evaluation of pain in children with cancer: narrative literature review
}

Evaluación del dolor en ninõs con cáncer: revisión de la literature narrativa

Valmor Arede Cordova Junior ${ }^{1 *}$, Silvestre Savino Neto ${ }^{1}$, Aurimery Gomes Chermont ${ }^{1}$, Creusa Barbosa dos Santos Trindade ${ }^{1}$, Suellen Patricia Sales da Costa Loureiro ${ }^{1}$, Heliana Helena de Moura Nunes ${ }^{1}$.

\section{RESUMO}

Objetivo: Descrever as escalas de avaliação da dor em crianças com câncer. Revisão bibliográfica: A dor é um dos principais e mais limitantes sintomas em crianças com câncer. Nessas crianças, muitas vezes a dor pode ser subestimada e subdiagnosticada. Avalia-la adequadamente é o primeiro passo para o manejo correto, contudo o uso errôneo das ferramentas disponíveis compromete o seu tratamento. Observou-se que o uso de ferramentas facilita a avaliação de dor em crianças, sendo o auto relato a medida mais fidedigna, o uso de figuras mostrou-se útil em crianças não alfabetizadas e as tecnologias em saúde aumentam a aderência das crianças ao processo de avaliação. Considerações finais: A avaliação da dor em crianças é complexa e fundamental para direcionar o tratamento, as escalas podem facilitar e sistematizar o processo. As escalas unidimensionais são as mais utilizadas na prática, sendo a intensidade da dor a dimensão mais comumente avaliada. Entretanto, as escalas multidimensionais são mais indicadas porque permitem uma compreensão mais abrangente da dor.

Palavras-chave: Medição da dor, Criança, Neoplasias.

\begin{abstract}
Objective: Describe pain assessment scales in children with cancer. Bibliographic review: Pain is one of the main and most limiting symptoms in children with cancer. In these children, pain can often be underestimated and underdiagnosed. Properly assessing it is the first step in the correct management, however the misuse of the available tools compromises its treatment. It was observed that the use of tools facilitates pain assessment in children, self-report being the most reliable measure, the use of figures proved to be useful in illiterate children and health technologies increase children's adherence to the process of evaluation. Final considerations: Pain assessment in children is complex and fundamental to direct treatment; therefore, it can change and facilitate the process. One-dimensional scales are the most used in practice, with pain intensity being the most common dimension assessed. However, multidimensional scales are more indicated because they can cause a more comprehensive understanding of pain.
\end{abstract}

Keywords: Pain measurement, Child, Neoplasms.

\section{RESUMEN}

Objetivo: Describir las escalas de evaluación del dolor en niños con cáncer. Revisión bibliográfica: El dolor es uno de los síntomas principales y más limitantes en los niños con cáncer. En estos niños, el dolor a menudo se puede subestimar e infradiagnosticar. Una adecuada valoración es el primer paso hacia una correcta gestión, sin embargo el mal uso de las herramientas disponibles compromete su tratamiento. Se observó que el uso de herramientas facilita la evaluación del dolor en los niños, siendo el autoinforme la medida más confiable, el uso de cifras demostró ser útil en niños analfabetos y las tecnologías de la salud aumentan la adherencia de los niños al proceso de evaluación. Consideraciones finales: La evaluación del dolor en los niños es compleja y fundamental para el tratamiento directo, las escalas pueden facilitar y sistematizar el proceso. Las escalas unidimensionales son las más utilizadas en la práctica, siendo la intensidad del dolor la dimensión más valorada. Sin embargo, las escalas multidimensionales son más adecuadas porque permiten una comprensión más completa del dolor.

Palabras clave: Dimensión del dolor, Niño, Neoplasias.

${ }^{1}$ Fundação Santa Casa de Misericórdia do Pará (FSCMPA), Belém - PA.

*E-mail:valmor_cordova@yahoo.com.br 


\section{INTRODUÇÃO}

Um dos principais males que acometem o paciente com câncer é a dor oncológica que se manifesta em todos os estágios do processo neoplásico. O tratamento inadequado da dor é comum em todo o mundo. Estima-se que no Brasil, cerca de 62 a 90\% dos pacientes com câncer apresentam algum tipo de dor, e que entre 25 a $45 \%$ dos pacientes com dor oncológica, têm sua dor inadequadamente controlada (CUNHA FF e REGO LV, 2015).

A dor em oncologia tem características peculiares. Para estes pacientes a dor tende a ser contínua, agravando-se na medida em que há evolução da doença neoplásica, levando o paciente a exaustão física e mental. Em crianças com câncer, a dor pode se evidenciar de diversas formas: causada pelo próprio tumor, pelo tratamento do tumor, pelos procedimentos, além de fatores incidentais como traumatismos e dores comuns na infância (MENOSSI MJ e LIMA RAG, 2004).

O número de casos de crianças com câncer aumenta continuamente, bem como de suas morbidades, e a dor é um sintoma muito comum em aproximadamente $80 \%$ das crianças com câncer, em regime de internação hospitalar, sendo que na fase terminal da doença a prevalência pode chegar até $90 \%$ (MELO LR e PETTENGILL MA, 2010).

A avaliação da dor nas suas várias dimensões, bem como dos efeitos que a acompanham, é uma tarefa essencial dos profissionais de saúde na prática clínica. A ausência de um processo que propicie uma avaliação adequada do quadro álgico na criança leva, muitas vezes, a não identificação e ao controle inadequado por parte da equipe de saúde. Além disso, práticas empíricas de avaliação geralmente levam à não valorização da dor, resultando em tratamento inadequado (CHOTOLLI MR e LUIZE PB, 2015).

O uso errôneo de instrumentos para mensurar e interpretar o processo doloroso na prática clínica pode comprometer a qualidade da assistência, uma vez que influenciam no correto diagnóstico e direcionamento da terapêutica. Portanto, é necessário que os profissionais de saúde conheçam os diversos instrumentos utilizados na avaliação da dor oncológica infantil, para que estejam capacitados para agir no tratamento da dor em crianças com câncer. Diante disso, este estudo objetiva realizar revisão narrativa sobre as principais ferramentas disponíveis para avaliação de dor em crianças com câncer.

\section{REVISÃO BIBLIOGRÁFICA}

A dor é uma experiência sensorial e emocional desagradável, multifatorial, subjetiva e deve ser avaliada, acompanhada e tratada respeitando seus aspectos subjetivos. Os profissionais de saúde devem ser capazes de identificar e avaliar o quadro álgico e promover o alívio do sofrimento doloroso. Para tal, há diversas ferramentas e técnicas de avaliação da dor e um vasto arsenal terapêutico medicamentoso e não medicamentoso (CHOTOLLI MR e LUIZE PB, 2015).

A dor em oncologia infantil tem propriedades específicas. Nestes pacientes a dor tende a ser contínua e com agravos de acordo com a evolução da doença neoplásica, levando o paciente a exaustão física, psíquica, mental e espiritual. A queixa de dor é bastante frequente entre pacientes com tumores avançados, tornando-se mais complexo diante da refratariedade do tratamento e das dificuldades de intervenções terapêuticas. Nestes casos avançados, frequentemente a dor é incapacitante e a abordagem terapêutica tem foco paliativo e nos alívios de sintomas, dentre os quais, a dor. (TORRITESI P e VENDRSCULO DMS, 1998).

Há diversas barreiras para o manejo da dor, dentre elas, falhas de comunicação, avaliação não rotineira da dor e dificuldades de entendimento da expressão da dor, podendo ser subestimada, subdiagnosticada, subtratada e associada a medo e sofrimento. Vários fatores podem afetar a percepção e a expressão da dor nas crianças, como: hereditariedade, gênero, maturação do sistema nervoso, cognição, personalidade, experiências dolorosas anteriores, gravidade da doença e a capacidade de autocontrole da dor. Sendo assim, é recomendável que a avaliação da dor reflita a complexidade dessa questão, incorporando componentes sensoriais, afetivos, cognitivos e interpessoais (CUNHA FF e REGO LV, 2015; PLUMMER K, et al., 2017). 
Em se tratando de crianças, a dor é influenciada pelo desenvolvimento cognitivo e capacidade de comunicação. Logo, a subjetividade da percepção e expressão da dor infantil é diretamente ligada à idade, portanto é necessária compreensão da equipe quanto a capacidade de comunicação da criança e acurácia quanto a escolha de ferramentas de avaliação (BUENO PC, et al, 2011).

A avaliação da dor infantil continua a ser um grande desafio, e as escalas de avaliação necessitam fornecer dados confiáveis e refletir adequadamente o quadro de dor da criança. Crianças maiores que 6 anos são capazes de fornecer informações de dor, através de escalas diretas como a escala visual analógica e numérica. Para crianças menores de 6 anos, as informações são obtidas por meio de observação. Já crianças acima de 10 anos, com capacidade cognitiva sem alterações, podem ser avaliadas mais detalhadamente através de escalas multidimensionais (MAREC-BERNARD P, et al., 2015).

Há três métodos para avaliação de dor em crianças: índices fisiológicos, avaliação comportamental e auto relato. As variações de indicadores fisiológicos, como de frequência respiratória e cardíaca, são usadas em neonatos; em crianças pré-escolar, na faixa etária até 6 anos, leva-se em conta as mudanças comportamentais, como choro e irritabilidade, uma vez que a capacidade cognitiva da criança é limitada para entender os comandos de avaliação da dor; a partir de cerca de 6 anos, o auto relato é considerado padrão ouro, caso não tenha limitações comunicativas, a criança é capaz de fornecer relato confiável de dor usando escalas de dor (MAREC-BERNARD P, et al., 2015).

O auto relato da dor permite realizar a avaliação pela perspectiva da criança, sendo mais fidedigno em demonstrar aquilo que a criança vivencia como experiência dolorosa. Desse modo, evita-se a subjetividade pela visão do que o profissional julga ser um quadro doloroso ou do que os familiares relatam ser dor na criança (MORETE MC e MINSON FP, 2010)

As escalas de dor são consideradas complementares, cada uma aborda de maneira diferente a dimensão avaliada. Estas ferramentas de avaliação de dor devem fornecer resultados reproduzíveis e confiáveis e são essenciais para fornecer dados objetivos, minimizando o viés subjetivo e interpretação do avaliador. As escalas podem ser unidimensionais ou multidimensionais. As escalas unidimensionais avaliam apenas uma única dimensão da dor, sendo a intensidade a dimensão mais comumente avaliada em pesquisas clínicas. A avaliação da intensidade permite analisar prevalência da dor e monitorar eficácia do tratamento. As escalas multidimensionais avaliam duas ou mais dimensões, como intensidade, localização, duração e fatores agravantes (BATALHA LMC, et al., 2015).

Entre as diferentes escalas, a que parece ser mais adequada à criança que ainda não foi alfabetizada ou não recebeu conhecimentos aritméticos é a escala das faces, que consiste numa técnica em que seis figuras de faces, do sorriso à aflição, são mostradas à criança. Essa escala foi validada para o uso em crianças maiores de três anos de idade e considerada de utilização fácil e rápida. Devido a criança está em fase de desenvolvimento cognitivo, muitos descritores de dor e palavras podem não fazer parte de seu vocabulário infantil, portanto o uso de figuras descritivas de dor facilita a avaliação e torna possível a construção do diagnóstico (ARAUJO CM, et al., 2012; STUDART PLM, et al., 2015).

A mensuração da intensidade da dor é utilizada como um parâmetro fundamental para a orientação terapêutica. A intensidade da dor é o critério mais comumente usados na prática clínica para quantificá-la e resulta da interpretação global dos aspectos sensitivos, emocionais e cognitivos que envolvem a experiência dolorosa. As escalas unidimensionais que avaliam a intensidade da dor permitem quantificar a experiência dolorosa e monitorizar as práticas de tratamento farmacológico quanto à sua eficácia, dentre estas, as mais utilizadas são a escala visual analógica (EVA) e a escala numérica verbal (ENV) (MELO LR e PETTENGILL MA, 2010; ARAUJO CM, et al., 2012).

Dufresne A, et al. (2010) a avaliaram a dor e ansiedade através da escala de faces e escala visual analógica, em crianças de 6 a 17 anos com câncer submetidas a procedimentos diagnósticos ou terapêuticos, nos seguintes momentos: antes de entrar na sala de procedimento, antes da aplicação de sedativos, após o procedimento e antes de sair da sala de procedimento. Foi também avaliado a percepção da dor das crianças pelos pais e médicos. Os resultados demonstraram que as avaliações dos pais e 
médicos foram imprecisas para determinar a dor das crianças, o que comprova que o auto relato das crianças é a medida mais fidedigna e que apesar de ser importante avaliar o comportamento dos pais, este pode ter um impacto negativo no sofrimento da criança. As escalas de face e visual analógica mostraram forte correlação, ou seja, para avaliação apenas da intensidade da dor sugere-se que o uso de apenas uma já seja suficiente, sendo que nessa idade acima de 06 anos deve-se dar preferência à EVA.

A intensidade dolorosa é uma das dimensões da dor mais avaliada na prática clínica e em pesquisas científicas. Dispõe-se de uma gama variada de escalas para avaliação de intensidade da dor. Dentre estas, a escala de Fichas de Poker, apesar de menos utilizada na rotina clínica, é uma ferramenta importante, simples e prática para avaliação da intensidade da dor. É uma medida de auto relato da dor para crianças de 4 a 13 anos, nesta avaliação a criança deve escolher de uma a quatro fichas vermelhas de poker, sendo que um representa pouca dor e quatro fichas representa a pior dor que a criança tenha sentido (VAN CLEVE L, et al, 2004).

Van Cleve L, et al. (2004) avaliaram a percepção e manejo da dor em crianças de 4 a 17 anos com leucemia aguda, a avaliação foi realizada em sete momentos distintos desde o diagnóstico à fase inicial de tratamento. A escala de fichas de poker foi usada para crianças de 4 a 7 anos, e constatou-se que a dor esteve presente em todos os momentos da avaliação e que a dor diminuía após manejo terapêutico, o que demonstra ser uma ferramenta eficaz para avaliar alívio da dor após intervenção terapêutica.

Fortier MA, et al. (2016) estudaram a dor através da escala de faces de dor revisada e o manejo da dor pelos pais de crianças entre 4 e 17 anos com câncer em tratamento domiciliar. Para otimizar a avaliação, o autor sugere que em crianças em tratamento domiciliar, seja utilizado avaliações de dor sincronizadas entre pais e crianças, pois os pais podem não ser capazes de detectar com precisão a dor. Sugere-se que sejam aplicadas escalas de avaliações de dor antes e após terapias analgésicas para avaliar eficácia do tratamento domiciliar de dor oncológica infantil.

Studart PLM, et al. (2015) realizaram entrevistas sobre dor com questões amplas, participaram da amostra crianças e adolescentes com câncer com idade acima de 8 anos, com objetivo de comparar os descritores de dor das crianças com os descritores do questionário de McGrill. Segundo os autores, as crianças usam termos concretos e figurativos para descrição da dor, o que reflete o seu estágio ainda de desenvolvimento cognitivo.

Percebe-se que os descritores (por exemplo, choque, pontada, agulhada, irradiada) são como metáforas para a expressão da dor, os quais permitem a criança organizar uma narrativa subjetiva da sua experiência dolorosa e transmitir a informação ao outro - profissional ou familiar; contudo nem sempre estes descritores fazem parte do vocabulário infantil, portanto no caso da avaliação em crianças é importante o uso de sinônimos dos descritores e de demonstrações de figuras dos descritores, o que torna possível avaliação mais fidedigna e facilita a construção do diagnóstico (STUDART PLM, et al., 2015).

Studart PLM, et al. (2015) ressaltaram a importância de se considerar na avaliação, o desenvolvimento cognitivo, idade da criança e capacidade de expressão da dor. É necessário que os instrumentos de avaliação multidimensional da dor utilizem figuras e imagens que façam alusão ao concreto, tanto para aspectos objetivos quanto subjetivos da avaliação e que estejam condizentes com o desenvolvimento da criança.

Dentre as ferramentas multidimensionais, há os diários de dor que permitem a captura de dados em momento real, minimizando o viés da recordação. Os diários eletrônicos de dor por meio de smartphone aumentam a adesão dos relatos de dor e melhoram sua validade, assim como, permitem avaliar a variabilidade da dor em um determinado período de tempo e otimizam a resposta do tratamento, por isso esse método tem se tornado padrão de avaliação de dor. Crianças a partir de 8 anos de idade já são capazes de usar o diário eletrônico e fornecer dados confiáveis. Devido ao avanço tecnológico de smartphones que se tornam corriqueiros em nossa rotina, estes tem sido cada vez mais utilizado como ferramenta de diário de dor (STINSON JN, et al., 2015).

Van Cleve L, et al. (2012) avaliaram 62 crianças com câncer (leucemia ou tumor solido estagio IV) entre 6 e 17 anos através da Escala de Avaliação de Sintomas Memoráveis, que consiste em escala 
multidimensional de 30 itens que avalia frequência e intensidade de um sintoma. Também, foi solicitado às crianças que localizassem a dor em um diagrama corporal através da marcação com caneta vermelha na área referida de dor. Os enfermeiros foram avaliados quanto a percepção da dor pelas crianças. Como resultado, as crianças relataram sentir dor em $56 \%$ do tempo, enquanto a dor foi documentada por enfermeiros em apenas $23 \%$, além disso, as crianças relatam intensidade da dor significativamente mais aguda do que os enfermeiros, estes dados ressaltam a importância do auto relato como padrão ouro de avaliação da dor em crianças e como a dor é subestimada por profissionais de saúde e também familiares.

O uso do diagrama corporal permitiu traçar a prevalência de locais de dor nas crianças, sendo a localização de dor mais frequente a cabeça com prevalência de $31 \%$, em seguida as localizações mais comuns de dor são abdominais (29\%), membros inferiores (26\%) e coluna lombar (24\%). Apesar dos esforços em manejar e reduzir a dor, a alta prevalência de dor demonstra que as escalas de localização e intensidade de dor foram eficazes em diagnosticar a dor, impedindo que este sintoma fosse subestimado (VAN CLEVE L, et al., 2012).

Stinson JN, et al. (2015) utilizaram um aplicativo eletrônico de avaliação multidimensional da dor em 92 crianças com câncer de 8 a 18 anos, as quais realizaram avaliação da dor no aplicativo duas vezes ao dia por três semanas. $O$ aplicativo permitia avaliação da intensidade, localização, duração da dor, interferência da dor na vida diária, entre outras dimensões do tratamento. $O$ aplicativo desenvolvido utiliza parâmetros de dor multidimensionais e simula um jogo com objetivos a ser alcançado pela criança, isto auxilia na fidelização da criança ao preenchimento do diário de dor. O uso de aplicativos na avaliação da dor oncológica pediátrica se mostrou uma ferramenta válida, viável e confiável.

Matziou V, et al. (2016) avaliaram a dor em crianças entre 3 e 18 anos, sendo 92 diagnosticadas com câncer e 159 diagnosticadas com distúrbios ortopédicos. As crianças foram avaliadas com o Questionário de dor pediátrico, instrumento multidimensional que avalia a presença e intensidade da dor, piora da dor na última semana e localização da dor. Nessa pesquisa, as crianças do sexo feminino e em idade pré-escolar apresentaram dor mais aguda. As crianças com distúrbios ortopédicos demonstraram dor mais intensa do que as crianças com câncer. As crianças menores, na faixa pré-escolar, apresentaram mais dor provavelmente devido menor capacidade de internalizar e manejar sozinha a dor, conforme a criança cresce, tende a verbalizar menos sua dor, outro fator que pode interferir também é o fato da criança menor ter o horizonte de consciência mais limitado o que pode dificultar sua expressividade.

Tutelman PR, et al. (2018) fizeram avaliação domiciliar de dor em crianças com câncer de 8 a 17 anos à distância através de aplicativo de celular par avaliar a eficácia do uso de aplicativos em um contexto real. Segundo as crianças, o aplicativo se mostrou útil para averiguação da dor e com potencial de penetração entre as crianças com dor oncológica, uma vez que as crianças participantes relataram que recomendariam seu uso para outras crianças, contudo o aplicativo foi avaliado negativamente para o tratamento da dor.

Apesar da tecnologia facilitar o cuidado e a promoção a saúde, ainda se encontram barreiras a serem superadas quando se sai do ambiente de pesquisa clínica para o contexto natural. Deve-se otimizar o tempo de avaliação, com processos e perguntas diretas, facilidade e automação da transmissão de dados em poucos passos (TUTELMAN PR, et al., 2018).

Em fases de remissão do câncer, a dor encontra-se bem controlada, portanto, nestes casos tende-se a ter um menor engajamento no uso de aplicativos para manejo da dor; deve-se direcionar seu uso, principalmente, para crianças em tratamento ativo. O uso de mecanismos de feedback e interação motivacionais nos aplicativos podem ser usados como mecanismos para adesão a longo prazo ao tratamento (TUTELMAN PR, et al., 2018).

Plummer K, et al. (2017) realizaram, auditoria do manejo de crianças em tratamento de câncer referente ao quadro de dor, monitorando as intervenções dos profissionais de saúde desde a avaliação até o tratamento da dor. Foram avaliadas crianças de 0 até 18 anos e foi coletado amostras durante 4 semanas, as quais geraram 258 episódios de atendimento. Este se tratou, na visão dos autores, do estudo mais abrangente sobre avaliação da dor infantil em oncologia dos últimos anos. No que se refere ao processo de avaliação, a dor estava presente em $57 \%$ dos atendimentos auditados, destes a causa da dor foi 
documentada em apenas $34 \%$ dos momentos, sendo a mucosite a principal causa de dor; já a localização da dor foi anotada em $56 \%$ dos registros, dentre os quais os mais comuns foram região perianal, boca e abdômen.

A intensidade da dor foi registrada em apenas $51 \%$ dos casos, dentre os casos registrados, a intensidade da dor foi principalmente leve. A ferramenta utilizada na avaliação da dor foi registrada em apenas 1 dos 258 momentos, na qual foi usada a escala de faces. Em nenhum registro foi coletado os impactos funcionais da dor em repouso e/ou movimento e sua relação com a intensidade da dor. Essa auditoria nos revela como no ambiente clínico, fora do controle rigoroso de uma pesquisa clínica, a avaliação da dor em crianças com câncer ainda é incompleta, inconclusiva e pouco eficaz em fornecer dados para o manejo adequado. Tais práticas de documentação podem estar relacionadas a ausência de diretrizes para avaliação da dor (PLUMMER K, et al., 2017).

\section{CONSIDERAÇÕES FINAIS}

Existem diversas escalas de avaliação da dor, contudo a aplicabilidade na prática clínica nem sempre é simples, pois a avaliação minuciosa da dor é complexa e longa. Dentre os instrumentos disponíveis, a ferramenta mais frequentemente utilizada são as escalas unidimensionais, a intensidade é a dimensão da dor mais comumente avaliada. Destaca-se a importância de utilizar instrumento multidimensional para avaliar a dor oncológica infantil, pois permitem uma abordagem mais ampla no manejo da complexidade da dor. Devido os diversos fatores que dificultam a utilização das escalas de avaliação da dor na rotina dos cuidados em saúde, tem crescido o uso das tecnologias em saúde que tornam mais acessíveis os instrumentos de avaliação de dor aos profissionais de saúde e ao alcance das crianças, a fim de otimizar avaliação da dor e consequentemente proporcionar a terapêutica adequada.

\section{REFERÊNCIAS}

1. ARAUJO CM, et al. Avaliação e tratamento da dor em oncologia pediátrica. Revista Médica de Minas Gerais, 2012; 22(Suppl.7): 22-31.

2. BATALHA LMC, et al. Pain assessment in children with cancer: a systematic review. Revista de Enfermagem Referência, 2015; 5(4): 119-127.

3. BUENO PC, et al. O manejo da dor em crianças com câncer: contribuições para enfermagem. Cogitare Enfermagem, 2011; 16(2): 226-31.

4. CHOTOLLI MR, LUIZE PB. Métodos não farmacológicos no controle da dor oncológica pediátrica. Revista Dor, 2015; 16(2): 109-13.

5. CUNHA FF, REGO LV. Enfermagem diante da dor oncológica. Revista Dor, 2015; 16(2): 142-45.

6. DUFRESNE A, et al. Do children undergoing cancer procedures under pharmacological sedation still report pain and anxiety? A preliminary study. Pain Medicine, 2010; 11(2): 215-223.

7. FORTIER MA, et al. Pain Buddy: A novel use of $m$-health in the management of children's cancer pain. Computers in Biology and Medicine, 2016; 76(1): 202-214.

8. MAREC-BERNARD P, et al. Pain scale: A shortened behavioral scale for assessment of prolonged cancer or postsurgical pain in children aged 2 to 6 years. Pediatric Hematology and Oncology, 2015; 32(5): 291-303.

9. MATZIOU V, et al. Perceptions of children and their parents about the pain experienced during their hospitalization and its impact on parents quality of life. Japanese Journal of Clinical Oncology, 2016; 46(9): 862-870.

10. MELO LR, PETTENGILL MA. Dor na infância: atualização quanto à avaliação e tratamento. Revista da Sociedade Brasileira de Enfermeiros Pediatras, 2010; 10(2): 97-102.

11. MENOSSI MJ, LIMA RAG. A dor da criança e do adolescente com câncer: dimensões de seu cuidar. Revista Brasileira de Enfermagem, 2004; 57(2): 178-182.

12. MORETE MC, MINSON FP. Instrumentos para avaliação da dor em pacientes oncológicos. Revista Dor, 2010; 11(1): 7480.

13. PLUMMER K, et al. Pain assessment and management in paediatric oncology: a cross-sectional audit. Journal of Clinical Nursing, 2017; 26(19-20): 2995-3006.

14. ROTHER ET. Revisão sistemática X revisão narrativa. Acta Paulista de Enfermagem, 2007; 20(2): 5-6.

15. STINSON JN, et al. Construct validity and reliability of a real-time multidimensional smartphone app to assess pain in children and adolescents with cancer. Pain, 2015; 156(12): 2607-2615.

16. STUDART PLM, et al. Descritores de dor presentes nas narrativas de crianças em tratamento oncológico. Estudos de Psicologia, 2015; 20(4): 241-250.

17. TORRITESI P, VENDRSCULO DMS. A dor na criança com câncer: modelos de avaliação. Revista Latino Americana de Enfermagem, 1998; 6(4): 49-55.

18. TUTELMAN PR, et al. The implementation effectiveness of a freely available pediatric cancer pain assessment app: A pilot implementation study. JMIR Cancer, 2018; 4(2): e10280.

19. VAN CLEVE $L$, et al. The pain experience of children with leukemia diagnosis during the first year after diagnosis. Nursing Research, 2004; 53(1): 1-10.

20. VAN CLEVE L, et al. Pain experience in children with advanced cancer. Journal of Pediatric Oncology Nursing, 2012;29(1):28-36

21. WHITTEMORE R. Analysis of integration in nursing science and practice. Journal of Nursing Scholarship, 2005; 37(3):2617. 